\title{
Ribavirin as a tri-targeted antitumor repositioned drug
}

\author{
ERICK DE LA CRUZ-HERNANDEZ ${ }^{1}$, JOSE LUIS MEDINA-FRANCO ${ }^{2,7}$, JAENAI TRUJILLO ${ }^{3}$, \\ ALMA CHAVEZ-BLANCO ${ }^{3}$, GUADALUPE DOMINGUEZ-GOMEZ ${ }^{3}$, ENRIQUE PEREZ-CARDENAS ${ }^{3}$, \\ AURORA GONZALEZ-FIERRO ${ }^{3}$, LUCIA TAJA-CHAYEB ${ }^{3}$ and ALFONSO DUEÑAS-GONZALEZ ${ }^{4-6}$ \\ ${ }^{1}$ Multidisciplinary Academic Division of Comalcalco, Juarez Autonomous University of Tabasco, Mexico; \\ ${ }^{2}$ Faculty of Chemistry, Department of Pharmacy, National Autonomous University of Mexico; ${ }^{3}$ Division of Basic Research, \\ National Cancer Institute of Mexico; ${ }^{4}$ Biomedical Research Institute, National Autonomous University of Mexico/ \\ National Cancer Institute of Mexico; ${ }^{5}$ ISSEMyM Cancer Center Toluca; ${ }^{6}$ On behalf of Tumor Study Group, Mexico
}

Received October 21, 2014; Accepted January 23, 2015

DOI: $10.3892 /$ or.2015.3816

\begin{abstract}
The aim of the present study was to demonstrate that ribavirin, a known inhibitor of eIF4E and inosine 5'-phosphate dehydrogenase (IMPDH), also inhibits histone methyltransferase zeste homolog 2 (EZH2). A computational searching revealed that ribavirin has a high structural similarity to 3-deazaneplanocin A (DZNep). The growth inhibitory effects of ribavirin as well as its effects upon epigenetic enzymes were evaluated in various cancer cell lines. siRNA assays were used to downregulate eIF4E, EZH2 and IMPDH to determine the contribution of these targets to the growth inhibitory effects of ribavirin. Ribavirin decreased EZH2 expression, inhibited histone methyltransferase activity and decreased H3K27 trimethylation. Ribavirin induced variable growth inhibition in a number of cell lines and downregulation of the targets, EZH2, eIF4E and IMPDH1 and 2 by siRNA led to comparable growth inhibition while no significant further reduction in viability was observed when siRNA transfected cells were treated with ribavirin. The results showed that ribavirin inhibits these cancer targets and should thus be studied for cancer therapy.
\end{abstract}

\section{Introduction}

Ribavirin, a synthetic nucleoside analogue (1- $\beta$-D-ribofuranosyl-1,2,4-triazole-3-carboxamide) is a widely used drug whose antiviral activity was described almost 40 years (1). Currently, ribavirin is almost solely used for hepatitis $\mathrm{C}$ infection (HCV)

Correspondence to: Dr Alfonso Dueñas-Gonzalez, Unidad de Investigación Biomédica en Cáncer, San Fernando 22, Tlalpan 14080, Mexico City, Mexico

E-mail: alfonso_duenasg@yahoo.com

Present address: ${ }^{7}$ Department of Research, Mayo Clinic, Scottsdale, AZ 85259, USA

Key words: ribavirin, drug repositioning, EZH2, eIF4E, inosine 5'-phosphate dehydrogenase, cancer in combination with interferon. This combination significantly increases sustained virological response rates even in patients with interferon resistance arising from $\mathrm{HCV}$-related factors (2). The knowledge gained on the molecular pathogenesis of cancer has led to considerable interest in repurposing non-cancer drugs against several malignancies (3). It was previously demonstrated that ribavirin and mycophenolic acid inhibit inosine 5'-phosphate dehydrogenase (IMPDH), the first enzyme specific for the de novo synthesis of guanosine monophosphate (GMP) (4). IMPDH encodes the rate-limiting enzyme in de novo guanine nucleotide biosynthesis, which is necessary for DNA and RNA synthesis. IMPDH has been linked to cell growth, differentiation and malignant transformation $(5,6)$. Two isoforms of IMPDH have been described: Type I is constitutively expressed in normal cells, whereas type II activity has been shown to be increased in proliferating and particularly malignant cells $(7,8)$. Thus, IMPDH has been considered an attractive target for cancer therapy (9).

On the other hand, Kentsis et al (10), demonstrated the direct binding of ribavirin to eIF4E in vitro at micromolar concentrations and an efficient competition with eIF4E:m7G mRNA cap binding in vitro and in vivo, which disrupts eIF4E:m7G functions in the transport and translation of eIF4E-regulated genes. These effects lead to downregulation of the oncogenic proteins, cell cycle arrest and suppression of the eIF4E-mediated transformation in vitro and in vivo (10). eIF4E is overexpressed in many human malignancies where it is typically a marker of poor prognosis. The function of this factor is controlled by interactions with protein cofactors together with many signaling pathways, including Ras, Mnk, Erk, MAPK, PI3K, mTOR and Akt. Borden and CuljkovicKraljacic suggest that $\sim 30 \%$ of cancer types have elevated eIF4E (11). In a recent proof-of-principle study on relapsed or refractory acute myeloid patients with subtypes M4 and M5 or other subtypes with high eIF4E levels, administered oral ribavirin at daily doses ranging from 1,000 to $1,800 \mathrm{mg}$ led to plasma ribavirin between 5 and $36 \mu \mathrm{M}$, produced 1 complete, 2 partial remissions, 2 blast response, and four stable diseases out of 11 evaluable patients. Best responses were observed at $\sim 28$ days (12). These results clearly support investigations on ribavirin as a cancer treatment. 
Beyond DNA methylation and histone deacetylase inhibitors, drugs able to block histone methylation are currently pursued as epigenetic anticancer drugs (13). Enhancer of zeste homolog 2 (EZH2), a core component of polycomb repressive complex 2 (PRC2), plays a role in transcriptional repression through $\mathrm{H} 3 \mathrm{~K} 27$ trimethylation, and is involved in various biological processes (14). It is well known that 3-deazaneplanocin A (DZNep), an inhibitor of S-adenosylmethionine-dependent methyltransferase targets the degradation of EZH2, leading to apoptosis in various malignancies, suggesting that EZH2 may be a new target for epigenetic treatment (15). The results of the present study showed that ribavirin is also an EZH2 inhibitor and that its antitumor effects stem from the inhibition of the IMPDH, eIF4E and EZH2 targets.

\section{Materials and methods}

Cell culture and ribavirin treatment. The breast (MCF-7, MDA-231 and MDA-436), brain (D54), cervical (HeLa), colon (SW-480) and prostate (PC3 and DU-145) cancer cell lines were obtained from the ATCC (Manassas, VA, USA) and cultured in Dulbecco's modified Eagle's medium (DMEM)/F12 medium supplemented with $10 \%$ fetal bovine serum (FBS) (both from Invitrogen Life Technologies, Carlsbad, CA, USA), and penicillin-streptomycin at $37^{\circ} \mathrm{C}$ in a humidified $5 \% \mathrm{CO}_{2}$ atmosphere. Ribavirin was obtained from Sigma (St. Louis, MO, USA), and dissolved in dimethyl sulfoxide (DMSO), stored at $-20^{\circ} \mathrm{C}$ and thawed prior to use. Stock solutions were thawed/ frozen $\leq 3$ times. Cell lines employed for the proliferation assay were treated with $10,15,20,25$ and $50 \mu \mathrm{M}$ for $96 \mathrm{~h}$. For the mRNA expression, and protein immunodetection, ribavirin treatment with $25 \mu \mathrm{M}$ was maintained for $72 \mathrm{~h}$.

Cell proliferation assay. Cells were seeded in 96-well microplates (Corning) at a density of $2 \times 10^{3}$ cells/well in $0.1 \mathrm{ml}$ of complete medium for $24 \mathrm{~h}$, and then treated for $96 \mathrm{~h}$ with ribavirin at concentrations of $10,15,20,25$ and $50 \mu \mathrm{M}$ with changes of medium containing ribavirin on alternate days. After $96 \mathrm{~h}$, the medium was aspirated and replaced for 10 min with $50 \mu \mathrm{l}$ of $0.75 \%$ crystal violet in $50 \%$ ethanol, $0.25 \% \mathrm{NaCl}$ and $1.75 \%$ formaldehyde solution. The cells were then washed with water, air-dried, and the dye was eluted with PBS $+1 \%$ sodium dodecyl sulfate (SDS) solution. The absorbance of crystal violet is proportional to the cell number and was assessed by dye absorbance measured at $570 \mathrm{~nm}$ on an automated microplate reader. All assays were performed in triplicate. The cytotoxic effect of each treatment was expressed as a percentage of cell proliferation relative to the untreated control cells (percentage of control) and was defined as (A570 nm treated cells/A570 nm non-treated cells) x 100 .

Computational search of approved drugs with a high structural similarity to DZNep. To identify in a systematic manner approved drugs with similar activity as DZNep, we carried out a rapid computational search of DrugBank, a database listing approved drugs (16). Using the well-established principles of similarity searching by two-dimensional fingerprints (17), we used the chemical structure of DZNep as a query and computed the similarity of each of 1,491 compounds listed in DrugBank using the Molecular Access System (MACCS) keys and the Tanimoto coefficient ${ }^{\mathrm{REF}}$ as implemented in Molecular Operating Environment (MOE), version 2011.10 (18,19). In general, compounds in DrugBank had an extremely low molecular similarity with DZNep (median and mean similarity values of 0.35 with a standard deviation of 0.12 ).

Flexible alignment in silico. The chemical structures of ribavirin and DZNep were constructed in MOE, version 2011.10 (18). The alignment of the two compounds was performed following the flexible alignment protocol implemented in MOE for small molecules. In this tool, the alignment is based on the internal strain and overlap of molecular features (20). For the flexible alignment we used default parameters including an iteration limit of 200, failure limit of 20, and an energy cut-off of 15 . Prior to the search, the charges were computed using the MMFF94x force field. The search was performed with the stochastic conformation search enabled. The top-ranked solution was used for analysis.

RNA isolation and quantitative expression assay. Total RNA was isolated using the PureLink RNA kit (Ambion-Life Technologies) according to the manufacturer's instructions. RNA purity and integrity were assessed by spectrophotometric analysis (NanoDrop 2000c; Thermo Scientific) and denaturing agarose gel. Total RNA $(1 \mu \mathrm{g})$ was used for cDNA synthesis with the SuperScript III First-Strand Synthesis SuperMix according to the manufacturer's instructions (Invitrogen Life Technologies). Quantitative PCR for amplification and detection of DNA was performed on the CFX96 thermocycler (Bio-Rad, Hercules, CA, USA). PCR was carried out in triplicate in a $10 \mu \mathrm{l}$ reaction volume with the SYBR-Green qPCR master mix (Bio-Rad), $0.35 \mathrm{nM}$ forward primer, and $0.35 \mathrm{nM}$ reverse primer. Data were analyzed using the $2^{-\Delta \Delta \mathrm{CT}}$ method and reported as the fold-change in gene expression normalized to the endogenous control gene $(G U S B)$ and relative to cells without treatment. The primers used were: GUSB, forward: 5'-CCTGTGACCTTTGTGAGCAA-3' and reverse: 5'-AAACC CTGCAATCGTTTCTG-3'; EZH2, forward: 5'-CAACACCAA GCAGTGCCCGTGC-3' and reverse: 5'-CCTGCCACGTCAG ATGGTGCC-3'; G9a, forward: 5'-CTCCGCTGATTTTCG AGTGTAA-3' and reverse: 5'-GTCGAAGAGGTAAGAATCA TCC-3'; LSD1, forward: 5'-GTGTCTCGTTGGCGTGCT-3' and reverse: 5'-CCCGCAAAGAAGAGTCGTG-3'; HDAC1, forward: 5'-GTTACACCATTCGTAACGTTGC-3' and reverse: 5'-CAGTCGCTGTTTGATCTTCTC-3; eif4E, forward: 5'-GCTTTGGTTAAAAATGGCTCA-3' and reverse: 5'-GAGACTGCCTTGCAATAAGAAG-3; Dnmt1, forward: 5'-TACCTGGACGACCCTGACCTC-3' and reverse: 5'-CGTT GGCATCAAAGATGGACA-3'; Dnmt3a, forward: 5'-TATT GATGAGCGCACAAGAGAGC-3' and reverse: 5'-GGGTGTT CCAGGGTAACATTGAG-3'; Dnmt3b, forward: 5'-GGCAAG TTCTCCGAGGTCTCTG-3' and reverse: 5'-TGGTACATGG CTTTTCGATAGGA-3'.

Protein extraction and immunoblot analysis. Whole-cell extraction was performed with lysis buffer containing $50 \mathrm{mM}$ Tris- $\mathrm{HCl} \mathrm{pH} 7.4,150 \mathrm{mM} \mathrm{NaCl}, 0.5 \%$ Nonidet P-40, $1 \mathrm{mM}$ EDTA, 1 mM phenylmethylsulfonyl fluoride (PMSF), $0.2 \mathrm{mM}$ $\mathrm{Na}_{3} \mathrm{VO}_{4}$ and a protease inhibitor cocktail (Sigma). Histones proteins were obtained by sulfuric acid extraction. Briefly, 

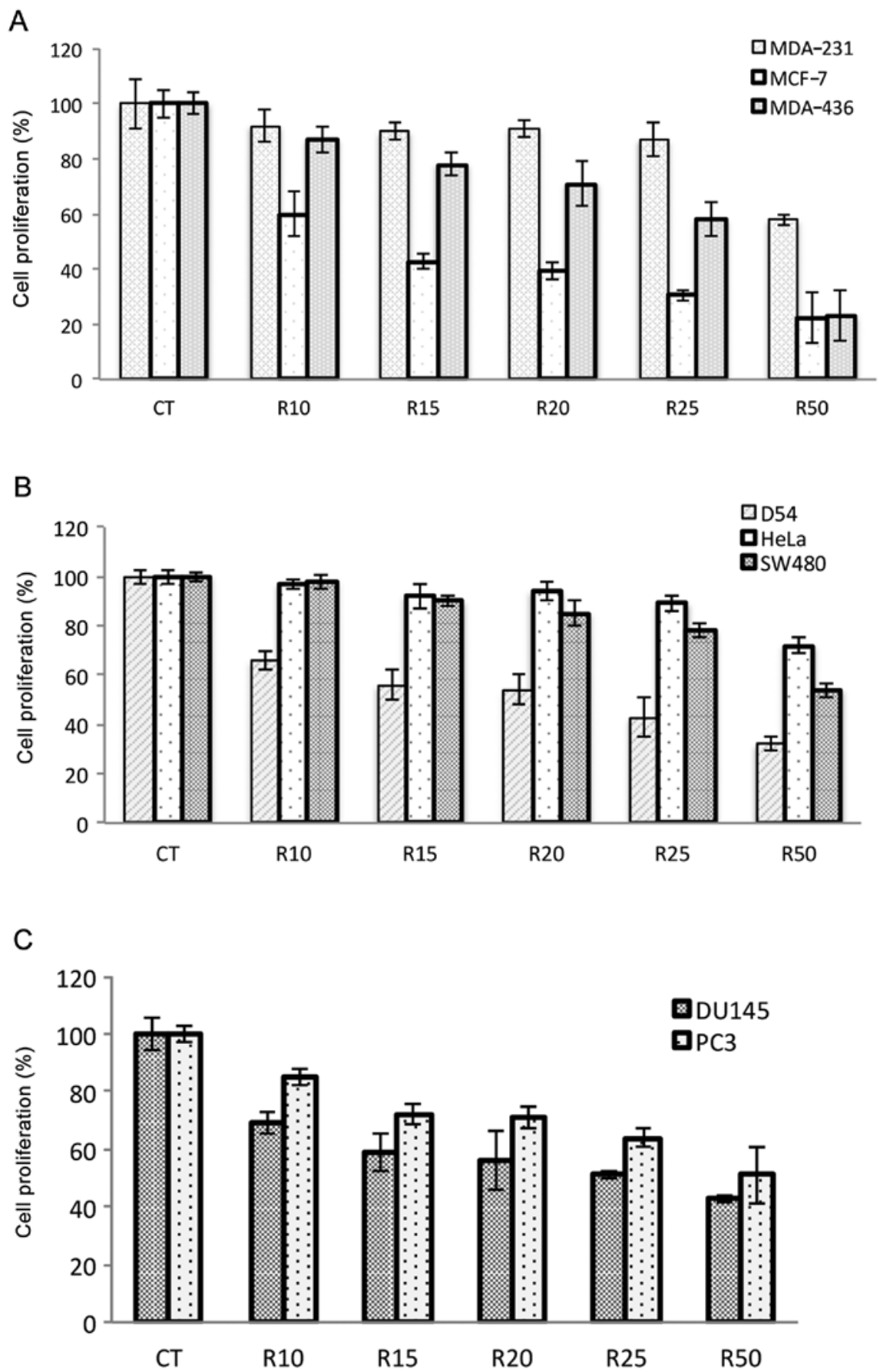

Figure 1. Growth inhibition by ribavirin. (A) Breast, (B) cervical, colon, and brain, and (C) prostate cancer cell lines were treated with different concentrations of ribavirin for $72 \mathrm{~h}$. Growth inhibition occurred in a cell line-dependent manner. Three independent experiments were performed (mean $\pm \mathrm{SD})$. Errors bars show SD.

the nuclear pellet was resuspended in $0.4 \mathrm{M} \mathrm{H}_{2} \mathrm{SO}_{4}$ for $4 \mathrm{~h}$, and acid-soluble proteins in a postcentrifugation supernatant $(10,000 \mathrm{xg}$ for $20 \mathrm{~min})$ were recovered by overnight precipitation with $20 \%$ trichloroacetic acid and centrifugation $(16,000 \mathrm{x} \mathrm{g}$ for $30 \mathrm{~min})$. The pellets containing the histone proteins were washed once in ice-cold acetone containing $1 \% \mathrm{HCl}$ and then washed once with ice-cold acetone. The pellet was dried under vacuum and stored at $-80^{\circ} \mathrm{C}$. The protein concentration was determined by the Bradford method, and the integrity of the histone proteins in the acid soluble extract was evaluated with Coomassie staining. Proteins were separated in an SDS-PAGE gel $10-18 \%$ according to the molecular weight of the studied protein, and transferred to a PVDF membrane (Bio-Rad). The membrane was incubated for $1 \mathrm{~h}$ with blocking solution (TBS/Tween-20, 5\% of non-fat milk), and incubated overnight with the specific primary antibody in blocking solution (dilution 1:1,000). The membranes were washed with TBS-T, and incubated $1 \mathrm{~h}$ with a peroxidase secondary antibody at a dilution of 1:1,000 and developed with chemiluminescence substrate cat. no. WBLUR0100 (Millipore, Billerica, MA, USA). Antibodies for EZH2 (cat. no. 36-6300) Invitrogen, G9a (cat. 07-551), histone 3 (cat. no. 06-755), H3K4me2 (cat. no. 07-030), H3K9me2 (cat. no. 07-212) and H3K27me3 (cat. no. 07-449) were obtained from Millipore. 
A<smiles>Nc1nccc2c1ncn2[C@@H]1C=C(CO)[C@@H](O)[C@H]1O</smiles>

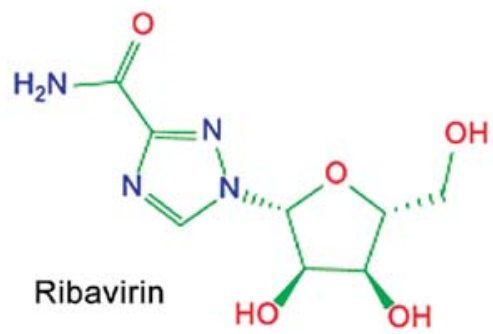

B

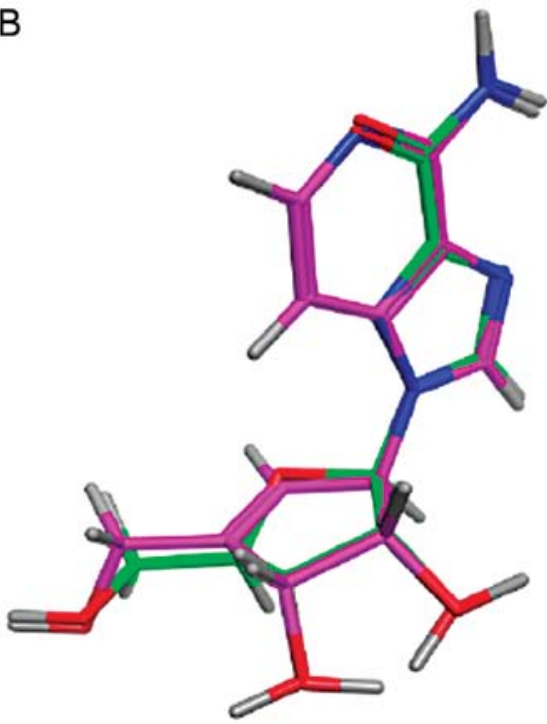

Figure 2. High structural similarity of ribavirin and DZNep. (A) Chemical structures of DZNep and ribavirin. (B) Result of the flexible alignment of the two compounds obtained with MOE. The image clearly shows the high structural similarity of ribavirin to DZNep in two- and three-dimensions. DZNep, 3-deazaneplanocin A; MOE, Molecular Operating Environment.

Inhibition of the histone methyltranferase activity. Histone methyltransferase activity/inhibition assay kit (Epigentek, New York, NY, USA) was employed to determine the inhibitory activity of ribavirin with HMT that specifically target histone $\mathrm{H} 3$ at lysine 27. Briefly, the nuclear extract was prepared from the MCF-7 cell line using the EpiQuik Nuclear Extraction kit (Epigentek), and incubated with ribavirin at 5, 10 and $15 \mu \mathrm{M}$. The percentage of ribavirin inhibition was calculated according to the level of $\mathrm{H} 3 \mathrm{~K} 27$ obtained in the control assay in the presence of $10 \mu \mathrm{g}$ of nuclear protein plus DMSO.

siRNA transfection assay. MCF-7 cells were seeded in 24-well microplate (Corning) at a density of $1.5 \times 10^{5}$ cells/well into $0.2 \mathrm{ml}$ of DMEM/F12 medium supplemented with 10\% FBS. After $24 \mathrm{~h}$, cells were transfected with the Lipofectamine RNAimax transfection reagent (Invitrogen-Life Technologies) and siRNA directed to EZH2 (4392420), eiF4E (4390771), IMPDH1 (4390771) and IMPDH2 (4390824) (Applied Biosystems-Life Technologies). Negative control was included as siRNA scramble (4390844) (Applied Biosystems-Life Technologies). Transfected cells were employed for the cytotoxicity assay as described above, and analyzed after $72 \mathrm{~h}$ with ribavirin treatment.

\section{Results}

Ribavirin inhibits cell growth. To investigate the growth inhibitory effects of ribavirin, the cell lines were exposed to different concentrations of ribavirin $(10-50 \mu \mathrm{M})$ and cell viability was analyzed at $72 \mathrm{~h}$. The results showed that the cell lines were inhibited in a dose-dependent manner although the extent of inhibition was cell line-dependent. The MCF-7 and MDA-436 breast cancer, DU145 prostate carcinoma and D54 glioma cell lines showed increased inhibition whereas the SW480 colon cancer, prostate PC3 and breast MDA-231 cells were less inhibited. HeLa cells showed minimal inhibition even at the highest dose of ribavirin (Fig. 1).
Ribavirin has a high structural similarity to DZNep. The search for similarity using DZNep as a query and after visual inspection of the 32 top-ranked compounds with a significantly increased similarity value compared to the observed mean value [i.e., mean plus 2 standard deviations (0.59)], ribavirin (similarity value of 0.70 ) was selected for additional threedimensional comparisons using flexible alignment. Following the 'principle of molecular similarity' (21) which states that, similar compounds have similar properties (with the exception of the so-called activity cliffs) (22) we performed a comparison in silico of the chemical structures of ribavirin and DZNep. Fig. 2A shows the two-dimensional chemical structures of the two molecules and the result of the top-ranked solution of the flexible alignment of both compounds obtained with Molecular Operating Environment software. Fig. 2B shows the almost perfect overlay of the pentose ring of ribavirin with the cyclopenten ring of DZNep. Furthermore, there was an excellent overlay in three dimensions of the 1,2,4-triazole carboxamide moiety of ribavirin with the 4-aminoimidazo[4,5-c]pyridine ring of DZNep leading to a perfect match of several nitrogen atoms, including the amino groups of the two molecules. The comparison of the two compounds clearly demonstrated that ribavirin has a high structural similarity to DZNep in the two- and three-dimensions. This analysis in silico further supported the hypothesis that the antiviral compound ribavirin has similar biological properties as DZNep.

Effects of ribavirin on epigenetic enzymes. To determine whether ribavirin exerts similar epigenetic effects compared to DZNep, quantitative RT-PCR was performed on MCF-7, PC3 and D54 cell lines to evaluate the effect of ribavirin upon several epigenetic enzymes. In MCF-7 cells ribavirin at $25 \mu \mathrm{M}$ led to a profound decrease in the level of EZH2 expression, while the effect was minor in PC3 cells and absent in D54 cells (Fig. 3A). Notably, a similar effect was observed for G9A histone methyltransferase expression. This downregulation was observed at the protein level as shown in Fig. 3B in MCF-7 
A

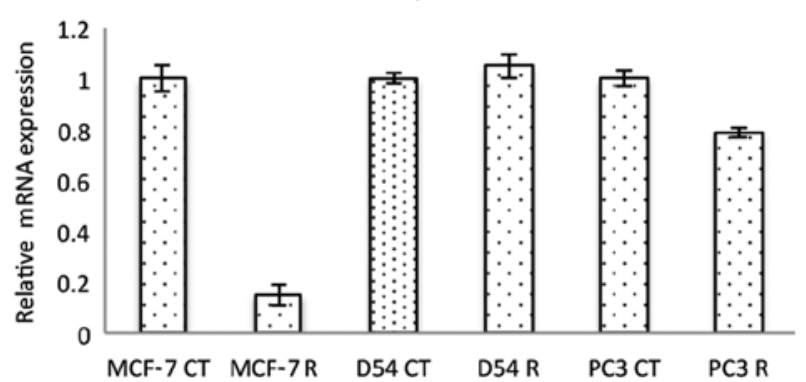

B

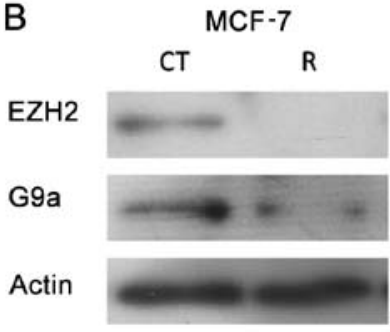

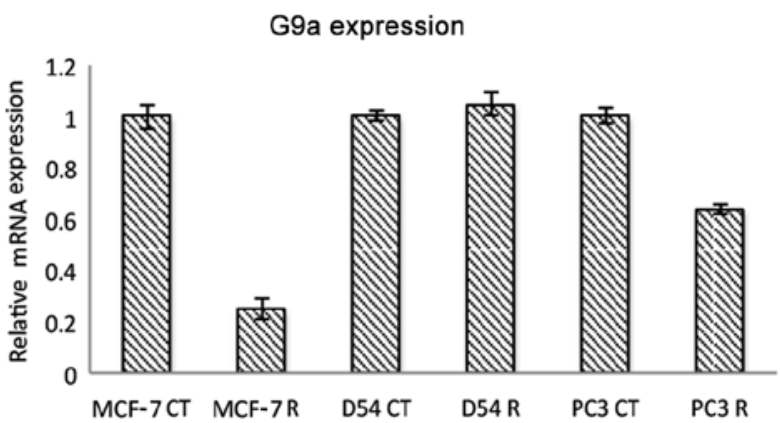

C CT $\quad 24 \mathrm{~h} \quad 48 \mathrm{~h}$ $72 \mathrm{~h}$

\section{$\mathrm{EZH} 2$}

Actin

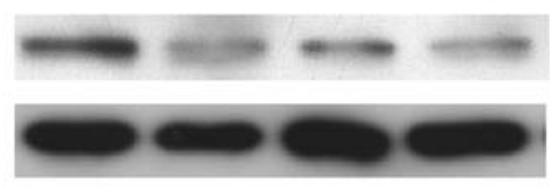

Figure 3. Inhibition of HMTs EZH2 and G9a. (A) Inhibition of EZH2 and G9a mRNA expression in breast and prostate cancer cell lines treated with ribavirin $(25 \mu \mathrm{M})$. (B) Western blotting of EZH2, and G9a methyltransferases (data were normalized according to GUS-B expression or $\beta$-actin protein levels, respectively). (C) Time-response curve of EZH2 protein in the MCF-7 cell line with ribavirin treatment. Errors bars show SD. EZH2, zeste homolog 2.
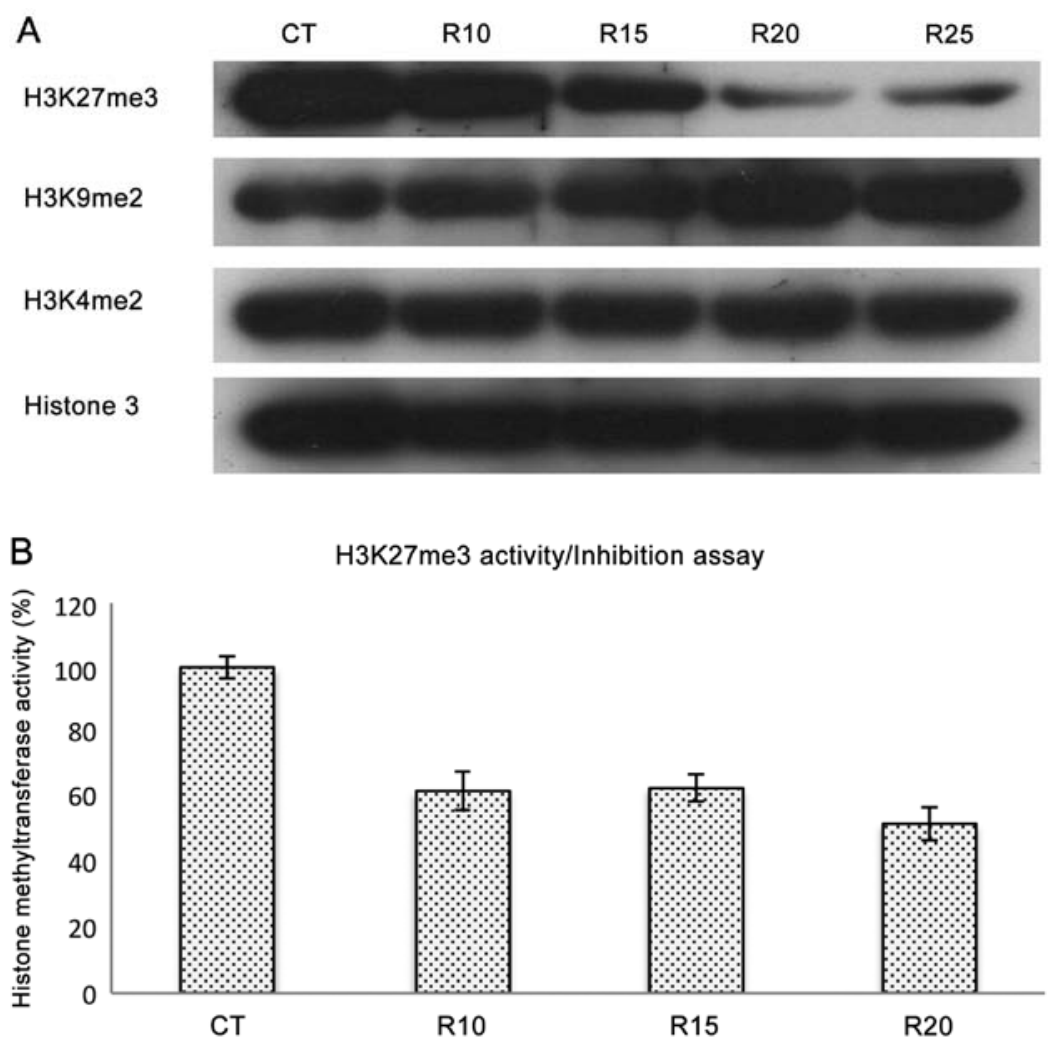

Figure 4. In vitro and in vivo dose-dependent inhibition of the repressive histone mark H3K27me3. (A) Immunoblot of H3K27me3 levels in MCF-7 cells treated with different doses of ribavirin. (B) In vitro histone methyltransferase activity (H3K27me3) in nuclear extracts from the MCF-7 cell line in the presence of DMSO (CT) or ribavirin (R). Error bars show SD.

cells treated with ribavirin at $25 \mu \mathrm{M}$ for $72 \mathrm{~h}$ and the effect appeared as early as $24 \mathrm{~h}$ (Fig. 3C). These data suggested that ribavirin inhibits histone methylation targets. Thus, the methylation levels of $\mathrm{H} 3 \mathrm{~K} 27$, H3K9 and $\mathrm{H} 3 \mathrm{~K} 4$ were analyzed by western blotting. Results in Fig. 4A shows that while ribavirin clearly decreased trimethylation of $\mathrm{H} 3 \mathrm{~K} 27$, no inhibition of methylation was observed for H3K9 and H3K4. To confirm these findings, an in vitro histone methyltransferase assay was 
A

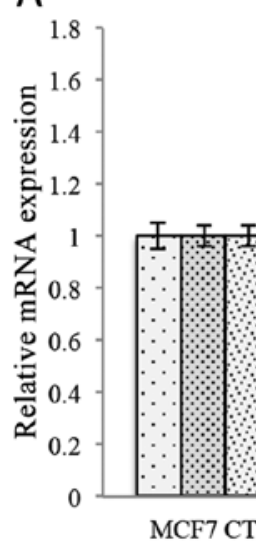

DNA methyltransferases

口Dnmtl expression

๑Dnmt3a expression

口Dnmt3b expression

B

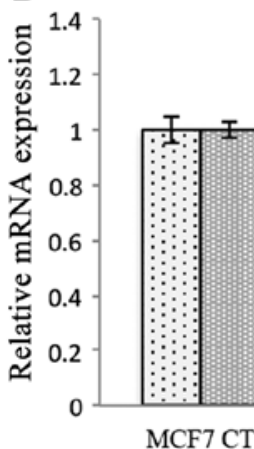

Histone-modifying enzymes

口HDACl expression 口LSD1 expression

Figure 5. Downregulation of DNA methyltranferases by ribavirin treatment. Expression of DNA methyltranferases, and enzymes associated with histone modification was analyzed by RT-qPCR in breast, brain and prostate cancer cell lines treated with $25 \mu \mathrm{M}$ ribavirin. Data were normalized according to $G U S B$ expression. (A) DNMT1 was inhibited only in MCF-7 cells, had no effect on D54 cells but was increased in PC3 cells. (B) Ribavirin did not induce changes in the expression of histone lysine demethylase 1. Errors bars show SD.

performed in MCF-7 cells treated with ribavirin. As shown in Fig. 4B, ribavirin at concentrations starting at $10 \mu \mathrm{M}$ showed a clear inhibition of the histone methyltransferase activity following H3K27. The expression of DNA methyltransferases was also evaluated following treatment with ribavirin. Fig. 5A shows that DNMT1 was inhibited only in MCF-7 cells, had no effect on D54 cells but was increased in PC3 cells. By contrast, DNMT3a was decreased to almost the same extent in the three cell lines, while DNMT3b was also decreased in three cell lines, although an increase was observed in MCF-7 cells. To explore other epigenetic enzymes that could be modified by ribavirin, we analyzed HDAC1, which was decreased in MCF-7 and D54 but not in PC3. Ribavirin did not induce changes in the expression of histone lysine demethylase 1 (LSD1) in these cell lines (Fig. 5B).

Target inhibition by ribavirin. To determine the extent that each ribavirin target contributed to the growth inhibitory effects of this drug on the cancer cell lines investigated, siRNA assays were used to downregulate eIF4E, EZH2 and both IMPDH1 and IMPDH2. As shown in Fig. 6A, each of the target genes were downregulated by $>50 \%$. Downregulation of EZH2 and eIF4E reduced cell viability almost to the same extent, with an $\sim 40 \%$ reduction as compared to the control. The extent of growth inhibition was slightly higher, reaching almost 50\% reduction for IMPDH1 and IMPDH2. No attempt was made to knock the targets down simultaneously, however, each individual target downregulation led to higher growth inhibition as compared to ribavirin at $25 \mu \mathrm{M}$. Notably, no significant further reduction in viability was observed when siRNA-transfected cells were treated with ribavirin.

\section{Discussion}

The results of the present study demonstrate that ribavirin at clinically achievable concentrations had growth inhibitory effects in vitro on a number of malignant cancer cell lines. Besides inhibiting eIF4E and IMPDH $(4,10)$ ribavirin led to downregulation of EZH2 at RNA and protein levels and inhibition of EZH2 enzyme activity and reduction of $\mathrm{H} 3 \mathrm{~K} 27$ methylation. These data support the repositioning of ribavirin as an antitumor drug, specifically in neoplasias whose growth is associated with alterations in these targets.

The realization that genetic alterations in histone methyltransferases occur in different types of tumor (23-25) has led to the development of epigenetic drugs beyond DNA methyltransferase (DNMTi) and histone deacetylase (HDACi) inhibitors. Concerning EZH2 histone methyltransferase, gain-of-function heterozygous point mutations in the SET-coding domain of the gene have shown to lead transcriptional repression via trimethylation of $\mathrm{H} 3 \mathrm{~K} 27$. In addition, the elevated levels of EZH2 have been associated with silencing of EZH2 target genes 

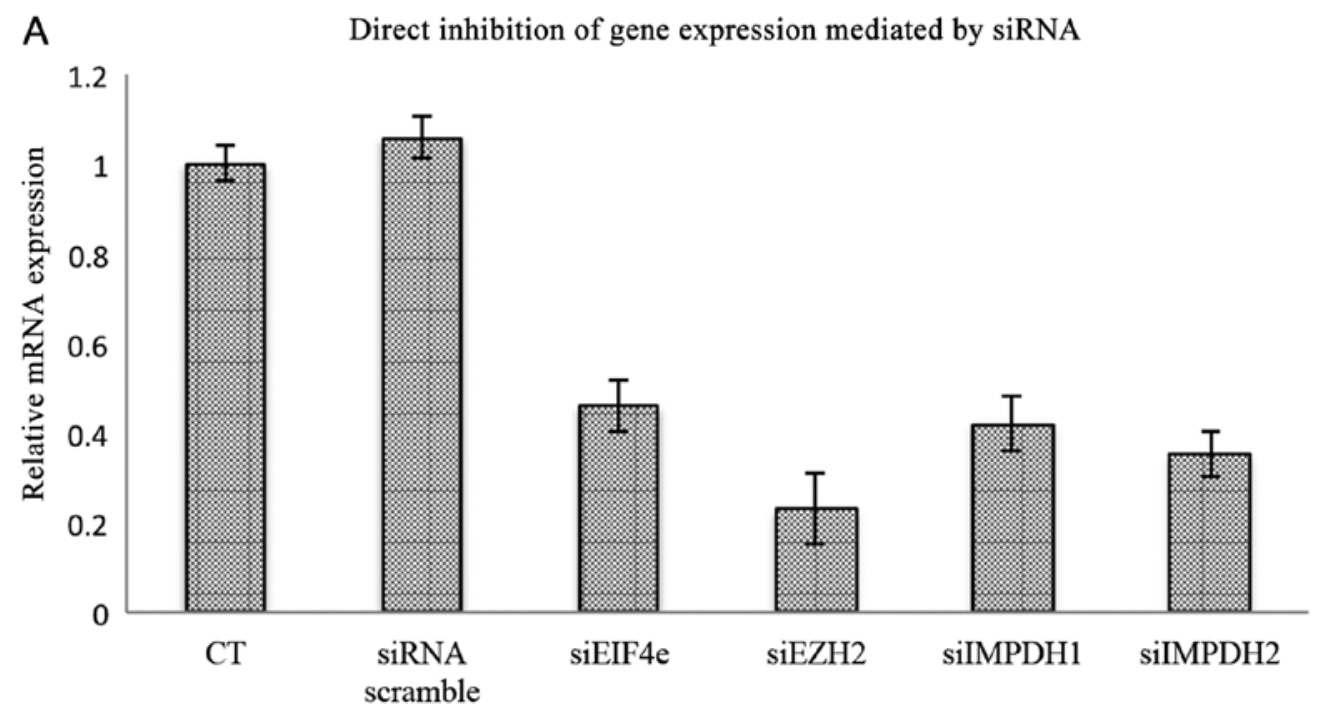

B Cell proliferation assay

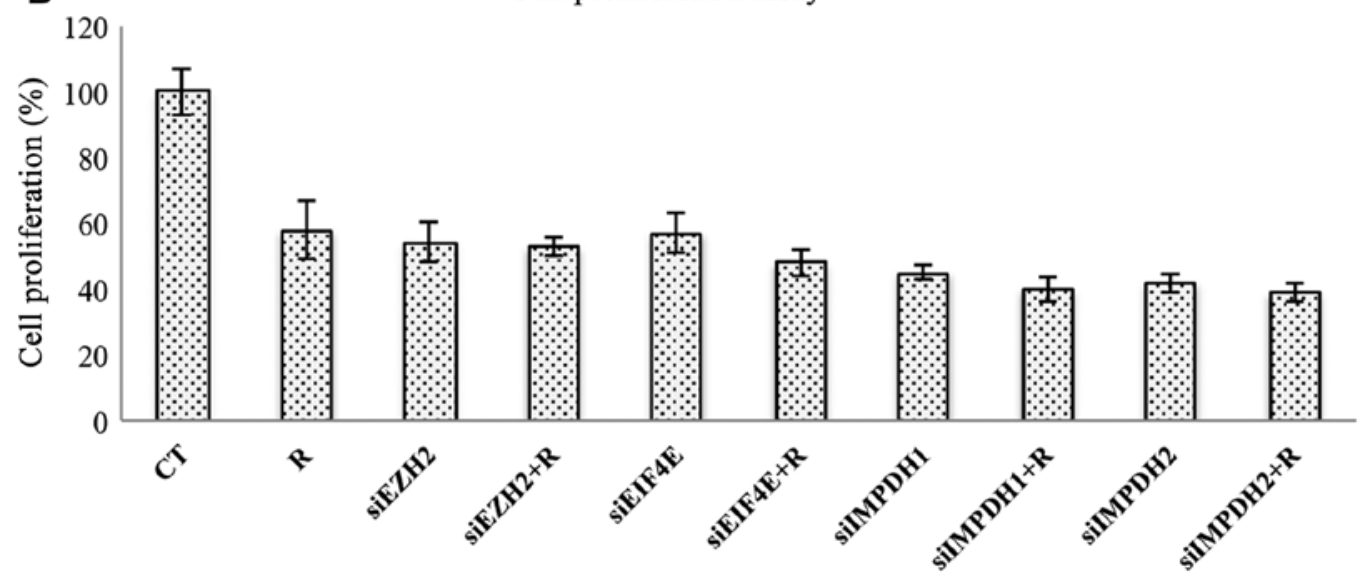

Figure 6. Downregulation of eIF4E, EZH2, MPDH1 and MPDH2 leads to growth inhibition. (A) siRNA transfection for EZH2, eIF4E and IMPDH1 and 2 in MCF-7 induced a decrease of $>60 \%$ in each gene. A scrambled sequence was the negative control. (B) Decreased expression of each of the targets led to reduced cell proliferation at the same or to a higher extent than ribavirin alone at $25 \mu \mathrm{M}$, which was not further increased when ribavirin was added. Errors bars show SD.

and poor prognosis in solid tumors such as breast, kidney and lung carcinomas (26-28). A number of EZH2 small-molecule inhibitors are in preclinical development, with E7438 already being in a phase I-II trial (unpublished data). Due to the proven tolerability and extensive use of ribavirin, the results from the present study suggest its rapid introduction into clinical trials for neoplasias with alterations at this oncogene.

DZNep is a potent inhibitor of S-adenosylhomocysteine (AdoHcy) hydrolase, which results in the accumulation of AdoHyc, which in turn causes by-product inhibition of S-adenosyl-L-methionine-dependent MTases (29). Initial studies have demonstrated a selective depletion of methylation targets on H3K27 and H3K20 (15). However, subsequent studies have shown that according to its proposed mechanism of action as a global inhibitor of SAM-dependent MTAses, DZNep inhibits histone methylation in a non-selective manner (30). Nevertheless, our results, although limited as only two additional methylation targets were analyzed, suggest that ribavirin is a potential selective histone methylation inhibitor. In particular, ribavirin induced a decrease in the trimethylation of H3K27. However, ribavirin did not induce changes in the methylation targets $\mathrm{H} 3 \mathrm{~K} 9$ and $\mathrm{H} 3 \mathrm{~K} 20$. A comprehensive analysis of histone methylation targets is needed to confirm whether ribavirin may be selected with $\mathrm{H} 3 \mathrm{~K} 27$ target.

As previously reported for DZNep, ribavirin also leads to the depletion of EZH2 RNA in MCF-7 although this effect is cell line-dependent as it was not observed in D54 glioma cells and prostate cancer PC3 cells. Of note, ribavirin also depletes H3K9 methyltransferase G9A in MCF-7 cells but not or only slightly in D54 and PC3, respectively. In this sense, DZNep has been shown to downregulate another H3K9 methyltransferase, SETDB1, in lung cancer cells (31). To demonstrate that ribavirin inhibits the methylation reaction at $\mathrm{H} 3 \mathrm{~K} 27$, nuclear extracts of MCF-7 cells treated with or without ribavirin between 10 and $15 \mu \mathrm{M}$ were assayed with a kit containing the substrate, the enzyme and adomet. The results in show an almost 50\% reduction in activity. As in mammals, EZH2 is the only enzyme that catalyzes di- and trimethylation at H3K27 (32). Our results suggest that ribavirin inhibits EZH2 in a similar manner to DZNep. Ribavirin treatment also affected the expression of some elements of epigenetic machinery in a variable manner depending on the cell line. In particular, the 
majority of changes were identified in MCF-7 cells, such as the reduction of DNMT1, DNMT3a, DNMT3b and HDAC1 but not LSD1. Notably, DNMT3b was consistently decreased in the three cell lines whereas LSD1 was not affected. To the best of our knowledge, no information exists on the effect of any EZH2 inhibitor on the expression of these enzymes. Since DZNep depleted cell proteins of the core PRC2 complex, as well as several other interacting proteins of this complex, such as Jarid2, HDAC1/2, Aebp2, Phf1, MTF2 and Phf19, it is suggested that significant interactions between polycomb proteins and other chromatin regulators may exist (33). In this sense, if DNMTs and HDACs also interact with the complex, then it is expected that they may be downregulated by ribavirin as a consequence of its depleting effect on EZH2; however, this remains to be demonstrated. Even for highly selective drugs, off-target effects are common. Thus, alternatively, the inhibition of IMPDH and eIF4E by ribavirin affects the expression of epigenetic machinery components by unknown mechanisms.

The present study on the antitumor effects of ribavirin as a tri-targeted therapy has some limitations. It is clear that growth inhibition while dose-dependent is also dependent on the cell line. Of the eight lines tested, MCF-7, MDA-436, D54 and DU-145 were the most sensitive. However, we have no information on the expression level of each of these putative targets on the model system. In a model of K562 leukemia cells, ribavirin induces differentiation and apoptosis while modulating the expression of $\sim 60$ out of 85 genes having roles in cell proliferation, purine biosynthesis, translation initiation, oncogenic signaling and cell survival (34). These effects are mediated through the modulation of key molecular and metabolic pathways but most likely result from the composed action of ribavirin on these three targets. In a study of 6 breast cancer cell lines investigating the antitumor effects of ribavirin as an eIF4E antagonist, it was shown that while ribavirin induces growth inhibition in all the cell lines, the variability in growth response observed did not correlate with eIF4E expression level (35). Our results on the individual downregulation of EZH2, eIF4E and IMPDH1 and IMPDH2 by $s i$ RNAs showed growth inhibition that varied between 36 and 55\%, being lower for eIF4E and higher for IMPDH2, which suggests that in this cell line viability is dependent on IMPDH2. Notably, in all the cases, the depletion of each of these genes led to higher growth inhibition as compared to ribavirin alone at $25 \mu \mathrm{M}$. However, ribavirin did not significantly increase the growth inhibition already achieved by downregulating the mRNA of the gene in any of the cases, suggesting that ribavirin is a tri-targeted agent. Other potential antitumor effects of ribavirin were not investigated in the present study. Recently, Kosaka et al, using an early transposon Oct4 and Sox 2 enhancer (EOS) system to select human prostate cancer cells expressing high levels of OCT4, a transcription factor that induces pluripotency in somatic cells, found that ribavirin reverses docetaxel resistance in prostatic cancer cells most likely by reprogramming cancer stem cells by downregulating OCT4 expression (36).

Our results are of relevance in the field of drug repositioning for cancer therapy as these three targets are commonly altered in cancers, supporting the current shift of drug identification from single- to multi-target drug development (37).
It is necessary however, to investigate which and what proportion of tumors share alterations in these targets and the manner in which their status correlates with sensitivity to ribavirin. However, a preclinical study to investigate whether non-Hodgkin lymphoma models with gain-of-function mutations in EZH2 are as sensitive to ribavirin as they are to the highly selective EZH2 inhibitor E7438 should be conducted. If these results are yielded a follow-up clinical study should be performed taking into account the known safety of ribavirin.

In conclusion, highly potent and selective small-molecule inhibitors of EZH2 are currently being investigated under the vision of 'rational drug design'. However, 'promiscuous' drugs that act on more than one relevant target for cancer biology such as ribavirin, should be studied to increase therapeutic armamentarium for cancer patients.

\section{Acknowledgements}

This study was supported by Fonsec CONACyT grant 161915, and UNAM PAPIIT grant IT206611.

\section{References}

1. Sidwell RW, Robins RK and Hillyard IW: Ribavirin: an antiviral agent. Pharmacol Ther 6: 123-146, 1979.

2. Shepherd J, Jones J, Hartwell D, Davidson P, Price A and Waugh N: Interferon alpha (pegylated and non-pegylated) and ribavirin for the treatment of mild chronic hepatitis $\mathrm{C}$ : a systematic review and economic evaluation. Health Technol Assess 11: 1-205, iii, 2007.

3. Dueñas-González A, García-López P, Herrera LA, MedinaFranco JL, González-Fierro A and Candelaria M: The prince and the pauper. A tale of anticancer targeted agents. Mol Cancer 7: 82,2008

4. Scheidel LM, Durbin RK and Stollar V: Sindbis virus mutants resistant to mycophenolic acid and ribavirin. Virology 158: 1-7, 1987.

5. Itoh O, Kuroiwa S, Atsumi S, Umezawa K, Takeuchi $\mathrm{T}$ and Hori M: Induction by the guanosine analogue oxanosine of reversion toward the normal phenotype of K-ras-transformed rat kidney cells. Cancer Res 49: 996-1000, 1989.

6. Jackson RC, Weber G and Morris HP: IMP dehydrogenase, an enzyme linked with proliferation and malignancy. Nature 256: 331-333, 1975.

7. Zimmermann A, Gu JJ, Spychala J and Mitchell BS: Inosine monophosphate dehydrogenase expression: transcriptional regulation of the type I and type II genes. Adv Enzyme Regul 36: 75-84, 1996.

8. Nagai M, Natsumeda Y, Konno Y, Hoffman R, Irino S and Weber G: Selective up-regulation of type II inosine 5'-monophosphate dehydrogenase messenger RNA expression in human leukemias. Cancer Res 51: 3886-3890, 1991.

9. Chen L and Pankiewicz KW: Recent development of IMP dehydrogenase inhibitors for the treatment of cancer. Curr Opin Drug Discov Devel 10: 403-412, 2007.

10. Kentsis A, Topisirovic I, Culjkovic B, Shao L and Borden KL: Ribavirin suppresses eIF4E-mediated oncogenic transformation by physical mimicry of the 7-methyl guanosine mRNA cap. Proc Natl Acad Sci USA 101: 18105-18110, 2004.

11. Borden KL and Culjkovic-Kraljacic B: Ribavirin as an anticancer therapy: acute myeloid leukemia and beyond? Leuk Lymphoma 51: 1805-1815, 2010.

12. Assouline S, Culjkovic B, Cocolakis E, Rousseau C, Beslu N, Amri A, Caplan S, Leber B, Roy DC, Miller WH Jr and Borden KL: Molecular targeting of the oncogene eIF4E in acute myeloid leukemia (AML): a proof-of-principle clinical trial with ribavirin. Blood 114: 257-260, 2009.

13. Zagni C, Chiacchio U and Rescifina A: Histone methyltransferase inhibitors: novel epigenetic agents for cancer treatment. Curr Med Chem 20: 167-185, 2013.

14. Simon JA and Lange CA: Roles of the EZH2 histone methyltransferase in cancer epigenetics. Mutat Res 647: 21-29, 2008. 
15. Tan J, Yang X, Zhuang L, Jiang X, Chen W, Lee PL, Karuturi RK, Tan PB, Liu ET and Yu Q: Pharmacologic disruption of Polycomb-repressive complex 2-mediated gene repression selectively induces apoptosis in cancer cells. Genes Dev 21: 1050-1063, 2007.

16. Wishart DS, Knox C, Guo AC, Cheng D, Shrivastava S, Tzur D, Gautam B and Hassanali M: DrugBank: a knowledgebase for drugs, drug actions and drug targets. Nucleic Acids Res 36: D901-D906, 2008

17. Willett P: Similarity-based virtual screening using 2D fingerprints. Drug Discov Today 11: 1046-1053, 2006.

18. Chemical Computing Group Inc., Montreal, Quebec, Canada: Molecular Operating Environment (MOE), version 2011.10. http://www.chemcomp.com.

19. Willett P, Barnard JM and Downs GM: Chemical similarity searching. J Chem Inf Comput Sci 38: 983-996, 1998.

20. Chan SL and Labute P: Training a scoring function for the alignment of small molecules. J Chem Inf Model 50: 1724-1735, 2010.

21. Bender A and Glen RC: Molecular similarity: a key technique in molecular informatics. Org Biomol Chem 2: 3204-3218, 2004.

22. Medina-Franco JL: Scanning structure-activity relationships with structure-activity similarity and related maps: from consensus activity cliffs to selectivity switches. J Chem Inf Model 52: 2485-2493, 2012.

23. Robinson G, Parker M, Kranenburg TA, Lu C, Chen X, Ding L, Phoenix TN, Hedlund E, Wei L, Zhu X, Chalhoub N, Baker SJ, Huether R, Kriwacki R, Curley N, Thiruvenkatam R, Wang J, Wu G, Rusch M, Hong X, Becksfort J, Gupta P, Ma J, Easton J, Vadodaria B, Onar-Thomas A, Lin T, Li S, Pounds S, Paugh S, Zhao D, Kawauchi D, Roussel MF, Finkelstein D, Ellison DW, Lau CC, Bouffet E, Hassall T, Gururangan S, Cohn R, Fulton RS, Fulton LL, Dooling DJ, Ochoa K, Gajjar A, Mardis ER, Wilson RK, Downing JR, Zhang J and Gilbertson RJ: Novel mutations target distinct subgroups of medulloblastoma. Nature 488: 43-48, 2012.

24. Ryan RJ and Bernstein BE: Molecular biology. Genetic events that shape the cancer epigenome. Science 336: 1513-1514, 2012.

25. Shih AH, Abdel-Wahab O, Patel JP and Levine RL: The role of mutations in epigenetic regulators in myeloid malignancies. Nature Rev Cancer 12: 599-612, 2012.

26. Kleer CG, Cao Q, Varambally S, Shen R, Ota I, Tomlins SA, Ghosh D, Sewalt RG, Otte AP, Hayes DF, Sabel MS, Livant D, Weiss SJ, Rubin MA and Chinnaiyan AM: EZH2 is a marker of aggressive breast cancer and promotes neoplastic transformation of breast epithelial cells. Proc Natl Acad Sci USA 100: 11606-11611, 2003.
27. Wagener N, Macher-Goeppinger S, Pritsch M, Hüsing J, Hoppe-Seyler K, Schirmacher P, Pfitzenmaier J, Haferkamp A, Hoppe-Seyler F and Hohenfellner M: Enhancer of zeste homolog 2 (EZH2) expression is an independent prognostic factor in renal cell carcinoma. BMC Cancer 10: 524, 2010.

28. Takawa M, Masuda K, Kunizaki M, Daigo Y, Takagi K, Iwai Y, Cho HS, Toyokawa G, Yamane Y, Maejima K, Field HI, Kobayashi T, Akasu T, Sugiyama M, Tsuchiya E, Atomi Y, Ponder BA, Nakamura Y and Hamamoto R: Validation of the histone methyltransferase EZH2 as a therapeutic target for various types of human cancer and as a prognostic marker. Cancer Sci 102: 1298-1305, 2011.

29. Chiang PK and Cantoni GL: Perturbation of biochemical transmethylations by 3-deazaadenosine in vivo. Biochem Pharmacol 28: 1897-1902, 1979.

30. Miranda TB, Cortez CC, Yoo CB, Liang G, Abe M, Kelly TK, Marquez VE and Jones PA: DZNep is a global histone methylation antagonist inhibitor that reactivates developmental genes not silenced by DNA methylation. Mol Cancer Ther 8: 15791588,2009

31. Lee JK and Kim KC: DZNep, inhibitor of S-adenosylhomocysteine hydrolase, down-regulates expression of SETDB1 H3K9me3 HMTase in human lung cancer cells. Biochem Biophys Res Commun 438: 647-652, 2013.

32. Margueron R, Li G, Sarma K, Blais A, Zavadil J, Woodcock CL, Dynlacht BD and Reinberg D: Ezh1 and Ezh2 maintain repressive chromatin through different mechanisms. Mol Cell 32: 503-518, 2008.

33. Simon JA and Kingston RE: Occupying chromatin: Polycomb mechanisms for getting to genomic targets, stopping transcriptional traffic, and staying put. Mol Cell 49: 808-824, 2013.

34. Kökény S, Papp J, Weber G, Vaszkó T, Carmona-Saez P and Oláh E: Ribavirin acts via multiple pathways in inhibition of leukemic cell proliferation. Anticancer Res 29: 1971-1980, 2009.

35. Pettersson F, Yau C, Dobocan MC, Culjkovic-Kraljacic B, Retrouvey H, Puckett R, Flores LM, Krop IE, Rousseau C, Cocolakis E, Borden KL, Benz CC and Miller WH Jr: Ribavirin treatment effects on breast cancers overexpressing eIF4E, a biomarker with prognostic specificity for luminal B-type breast cancer. Clin Cancer Res 17: 2874-2884, 2011

36. Kosaka T, Nagamatsu G, Saito S, Oya M, Suda T and Horimoto K: Identification of drug candidate against prostate cancer from the aspect of somatic cell reprogramming. Cancer Sci 104: 1017-1026, 2013.

37. Medina-Franco JL, Giulianotti MA, Welmaker GS and Houghten RA: Shifting from the single to the multitarget paradigm in drug discovery. Drug Discov Today 18: 495-501, 2013. 